\title{
Effet de la Culpabilité Groupale Ressentie sur le Soutien a la Réparation de L'oppression a L'égard de L'exogroupe : Le cas des Anglophones du Cameroun
}

\author{
Messanga Gustave Adolphe, \\ Maître de Conférences de Psychologie Sociale, \\ Université de Dschang, Cameroun \\ Ekango Nzekaih Hermann, Kevin \\ Doctorant en Psychologie Sociale, Université de Dschang, Cameroun \\ Npiane Ngongueu Sonia, \\ Doctorante en Psychologie Sociale, Université de Dschang, Cameroun
}

Doi:10.19044/esj.2020.v16n10p135 URL:http://dx.doi.org/10.19044/esj.2020.v16n10p135

\section{Résumé}

La littérature portant sur la relation entre les émotions collectives et le soutien à la compensation de l'oppression intergroupe révèle que les émotions collectives ressenties par les membres d'un groupe sont à l'origine de leur réaction à l'égard des injustices commises par l'endogroupe à l'endroit d'un exogroupe victime (Allpress et al., 2010; Licata \& Klein, 2010). En contribution à cette littérature, la présente recherche propose que dans le cadre des relations intergroupes linguistiques en contexte camerounais, la culpabilité groupale ressentie par les membres de la communauté linguistique francophone détermine leur soutien à la réparation de l'oppression à l'égard de la minorité linguistique anglophone. Les participants sont 184 étudiants francophones des deux sexes, fréquentant diverses facultés de l'Université de Dschang (Cameroun), et âgés entre 18 et 50 ans $(M=21.91$; É.-T.=4.59). Ils ont répondu à deux échelles, dont l'une mesure la culpabilité groupale ressentie (Allpress et al., 2010) et l'autre le soutien à la réparation de l'oppression à l'égard de la minorité linguistique anglophone, dont les items sont formulés sur la base de la littérature relative à la réparation des violences intergroupes (Staub, 2008). Les données collectées à l'issue de cette recherche apportent un soutien empirique à l'hypothèse formulée. En effet, la culpabilité groupale ressentie par les participants les pousse à soutenir la réparation de l'oppression à l'égard de l'exogroupe linguistique anglophone.

Mots Clés : Emotion collective, culpabilité groupale ressentie, soutien à la réparation de l'oppression intergroupe, Anglophones, Francophones 


\title{
Effect of Feelings of Guilt on Support for Oppression Reparation for Outgroup: The Case of Anglophones of Cameroon
}

\author{
Messanga Gustave Adolphe, \\ Maître de Conférences de Psychologie Sociale, \\ Université de Dschang, Cameroun \\ Ekango Nzekaih Hermann, Kevin \\ Doctorant en Psychologie Sociale, \\ Université de Dschang, Cameroun \\ Npiane Ngongueu Sonia, \\ Doctorante en Psychologie Sociale, \\ Université de Dschang, Cameroun
}

\begin{abstract}
Literature on the relationship between group emotions and support for reparation drawn from intergroup oppression reveals that feelings of guilt by group members originate from their reaction towards injustices committed by the ingroup vis-à-vis the outgroup (Allpress et al., 2010; Licata \& Klein, 2010). As contribution to this growing body of knowledge, this study suggests that in the context of linguistic intergroup relations in the Cameroonian context, group guilt felt by members of the francophone linguistic community determines their support for oppression reparation towards the Anglophone linguistic minority. Participants constituted 184 francophone students of both sexes in different Faculties of the University of Dschang (Cameroon), aged between 18 and 50 years $(M=21.91$; S.D. $=4.59)$. Participants responded to two scales: measures of feelings of group guilt (Allpress et al., 2010) and measure of oppression reparation constructed on the basis of literature on reparation for intergroup violence (Staub, 2008). Analysis of data collected from the study provided empirical support for the hypothesis. Therefore, it was observed that feelings of group guilt of participants urged them to support oppression reparation for the Anglophone linguistic outgroup.
\end{abstract}

Keywords: Group emotion, group guilt, intergroup oppression, reparation, Anglophones, Francophones 


\section{Introduction}

Plusieurs études sur les émotions individuelles et collectives se basent sur les modèles évaluatifs (Fischer \& Manstead, 2016 ; Lazarus, 1991 ; WeissKlayman et al., 2020). Pour ces travaux, l'expression d'une émotion est initiée par l'évaluation des situations, actes ou évènements basés sur des cognitions individuelles (buts, sentiments et désirs). Selon Parkinson (2001), ces modèles peuvent également inclure l'aspect social. Il s'agit précisément de la prise en considération des aspects sociaux dans le processus évaluatif. L'expression des émotions individuelles ou collectives est ainsi influencée par des règles et valeurs sociales; d'où le lien qu'on peut établir entre émotion individuelle et émotions et évaluations intergroupes (Manstead \& Fischer, 2001 ; Rimé, 2009). Précisément, la théorie des émotions intergroupes stipule que les individus éprouvent des émotions à propos de leur groupe d'appartenance et adoptent des comportements sociaux distincts de ce qu'ils ressentent personnellement (Mackie et al., 2000 ; Mackie et al., 2004). L'identification groupale est pertinente ici, car elle permet à l'individu d'éprouver des émotions collectives liées à un événement, même s'il n'est pas personnellement impliqué dans l'événement collectif ou si celui-ci appartient à l'histoire de son groupe (Smith, 1993). L'expérience émotionnelle dépend donc de l'appartenance groupale et fonctionne à la fois aux niveaux groupal et individuel. En d'autres termes, par le processus de catégorisation et d'identification sociale, l'émotion individuelle converge vers l'expérience émotionnelle vécue par le groupe.

La littérature portant sur les relations intergroupes s'intéresse à la réaction émotionnelle des membres d'un groupe, suite aux violences et atrocités perpétrées à l'encontre des membres d'un exogroupe, notamment en lien avec des événements comme l'holocauste, l'exploitation des populations autochtones et le passé colonial (Iyer \& Leach, 2008 ; Licata \& Klein, 2005). Les résultats de recherche révèlent que la culpabilité et la honte collectives sont les réponses émotionnelles les plus largement liées à ces événements (Licata \& Klein, 2005 ; 2010). Ces deux émotions découlent de la perception que l'endogroupe est l'auteur d'une action injuste, débordant les limites morales. Elles traduisent des états émotionnels négatifs qui mettent les individus en situation d'inconfort. La honte et la culpabilité collectives sont également liées à des intentions, des actions ou des comportements sociaux à l'endroit des membres de l'exogroupe (Baumeister et al., 1994 ; Brown \& Čehajić, 2008 ; Iyer et al., 2007 ; Lewis, 1971 ; Tangney \& Fischer, 1995). Elles engendrent des états spécifiques qui incitent les individus à prendre des mesures prosociales à l'égard du groupe d'appartenance ou des groupes de non appartenance impliqués dans l'événement (Iyer \& Leach, 2008 ; Thomas et al., 2009). 
Tout comme les émotions conscientes de soi, les actions sociales que les individus entreprennent dépendent fortement de la nature de l'émotion vécue (Tiedens \& Leach, 2004). En d'autres termes, la nature de l'émotion ressentie détermine la qualité des relations intergroupes. La littérature existante révèle, en effet, qu'il y a des liens entre la culpabilité collective et le soutien des populations à la réparation des tensions intergroupes (Iyer et al., 2003 ; Leach et al., 2006). Par exemple, Allpres et al. (2010) affirment que la honte et la culpabilité collectives affectent positivement le soutien des membres des groupes d'ex-colonisateurs aux excuses et à la compensation à l'égard des victimes de leur colonisation. Dans la même veine, Doosje et al. (1998) indiquent qu'éprouver de la culpabilité collective a des effets positifs sur le processus de réconciliation, à l'instar du soutien aux politiques de réparation ou aux excuses publiques. C'est dans cette perspective que se situe la présente étude, qui s'intéresse aux stratégies de réparation de l'oppression intergroupe.

Aucune société n'est un ensemble homogène, uniforme ou définitif. En effet, toute société est constituée de groupes sociaux dont les valeurs, les intérêts et les objectifs ne coïncident pas nécessairement. La cohabitation de deux ou plusieurs entités groupales est donc susceptible de conduire à des points de vue divergents; ce qui est déjà un premier pas vers un conflit potentiel. Dans cette logique, Nadler et Shnabel (2015) indiquent que tout conflit s'inscrit dans le modèle classique rationnel des interactions entre groupes. Selon ce modèle, le conflit découle d'une compétition lorsque des groupes luttent pour acquérir des ressources importantes et rares (Sherif, 1958). En d'autres termes, il est attribuable à des désaccords liés au processus de répartition des ressources rares et convoitées. C'est pourquoi, dans la perspective du règlement des conflits (Kelman, 2008), on se focalise sur les positions contradictoires, qui donnent lieu à des négociations distributives basées sur les moyens de partager les ressources en question. L'accent mis sur les intérêts sous-jacents des parties aboutit à des négociations intégratives avant le partage (Cross \& Rosenthal, 1999).

D'après Deutsch (1973) et Kelman (2008), dans le domaine de la résolution des conflits, l'accord sur la répartition des ressources n'est pas suffisant. En effet, une compréhension complète du conflit entre groupes nécessite de changer les relations entre les adversaires et de prendre en compte leurs besoins et sentiments (besoins de justice et d'égalité, sentiments de victimisation ou de violence). Ainsi, la réconciliation entre groupes est un processus qui mène à une fin stable du conflit et repose sur des changements dans la nature des relations conflictuelles entre les adversaires. Selon Nadler (2012) et Nadler et Shnabel (2015), la réconciliation ou la réparation de l'oppression révèle trois axes liés, mais différents. Il s'agit des axes structurel, relationnel et identitaire. 
L'axe structurel, particulièrement pertinent dans les contextes où les parties en conflit appartiennent à la même société, considère le but de la réconciliation comme la transformation des relations de pouvoir entre les groupes favorisés et défavorisés dans une structure sociale fondée sur l'égalité (Rouhana, 2004). À titre d'exemple, l'analyse des processus de réconciliation post-apartheid en Afrique du Sud indique qu'une réconciliation stable entre les Noirs et les Blancs dépend des changements structurels dans le sens d'une plus grande égalité raciale (Du Toit \& Doxtader, 2010). L'axe relationnel fait la promotion d'une confiance accrue et de relations intergroupes plus positives (Kriesberg, 2007). Les programmes interpersonnels, ainsi que les efforts de renforcement de la communauté et de la confiance entre les anciens adversaires en sont des exemples concrets. Quant à l'axe identitaire, il suggère que les conflits menacent l'identité des parties impliquées; d'où la poursuite du conflit (Kelman, 2008). Par exemple, lorsqu'un groupe a été vaincu et est victimisé, ses membres peuvent se sentir humiliés et chercher à se venger pour tenter de restaurer son identité et sa dignité (Lindner, 2006). En revanche, lorsque le groupe a commis des actes violents graves contre les membres d'un exogroupe, la nécessité de maintenir son identité positive peut amener ses membres à se désengager de ces actes immoraux en niant toute responsabilité (Bandura, 1999). Cette posture empêche la fin du conflit. Il en découle que cet axe considère la réconciliation comme un moyen de rétablir l'identité sociale préalablement fragilisée par les conflits ou les violences intergroupes.

Dans l'ensemble, ces trois axes d'analyse suggèrent que la réconciliation entre groupes induit des relations positives et de confiance entre les anciens adversaires, qui jouissent d'une identité sociale sûre et interagissent de manière équitable dans un environnement social (Nadler, 2012). Toutefois, il est important de noter que la distinction entre les axes structurel, relationnel et identitaire n'est faite que dans un souci de clarté conceptuelle car, en réalité, ils sont interdépendants. En soutien à cette perception, les recherches menées en Irlande du Nord (Tam et al., 2008) et en ex-Yougoslavie (Cehajic et al., 2008) révèlent que les relations chaleureuses entre les anciens belligérants sont associées à la volonté de pardonner aux auteurs des actes commis dans le passé (un aspect de la réconciliation lié à l'identité). Ainsi, les trois aspects de la réconciliation opèrent ensemble pour faciliter une orientation positive générale vers l'autre, qui soit solide et durable.

Les Commissions "vérité et réconciliation" (CVR) en Afrique du Sud et ailleurs (Avruch \& Vejerano, 2002), et les excuses publiques présentées par les dirigeants politiques à un groupe autrefois victime de traitements discriminatoires et/ou violents (Blatz et al., 2009) sont quelques illustrations des efforts déployés pour désarmer les barrières émotionnelles liées aux conflits et améliorer les relations intergroupes. Les CVR et les efforts se 
situant dans le même sillage sont sous-tendus par l'idée que la conciliation des intérêts divergents des groupes ne suffit pas pour assurer la fin des conflits et une paix durable. Pour y parvenir, il faut adresser directement et ouvertement la question du passé injuste, inégal et douloureux qui a créé des barrières émotionnelles et perceptuelles de la victimisation, la culpabilité, la méfiance et la peur (Barkan, 2001). Une autre manifestation de la résolution des conflits est le développement de la résolution alternative dans la théorie et la pratique juridiques (Boyes-Watson, 2008). Contrairement à l'emphase plus traditionnelle qui consiste à punir le malfaiteur (Whitman, 2003), la théorie et la pratique juridique cherchent à mettre fin au conflit en guérissant les relations rompues entre les parties ; désarmant ainsi les émotions négatives de chacune envers l'autre (Braithwaite, 2002). Ces éléments sont-ils applicables à la crise sécessionniste en cours dans les régions anglophones du Cameroun ?

La triple colonisation européenne subie par le Cameroun lui a donné une certaine spécificité (Abwa, 2000). En effet, l'histoire coloniale de ce territoire, sous protectorat allemand depuis 1884, révèle qu'il a été placé sous le mandat de la Société Des Nations (SDN) à l'issue de la première guerre mondiale ; un conflit qui s'est achevé par la défaite de l'Allemagne. La SDN en a confié l'administration à la France et à l'Angleterre jusqu'à l'indépendance. Comme l'indique la Milner-Simon Boundary Declaration de 1916, quatre cinquième (4/5) du territoire, comprenant trois quarts (3/4) de la population ont été attribués à la France, tandis qu'un cinquième du territoire $(1 / 5)$, comprenant un quart (1/4) de la population a été attribué à l'Angleterre. Dès lors, ces deux puissances coloniales ont entrepris de façonner les populations locales à leur image, en procédant à une dégermanisation systématique. Ainsi, elles ont imposé leurs propres cultures respectivement sur les deux parties du territoire placées sous leur administration. Il en résulte qu'après quarante-quatre ans (44) d'administrations anglaise et française (1916-1960), les divergences culturelles entre les deux Cameroun sont profondes. Elles sont observables notamment dans les domaines de l'éducation, de l'administration locale ou de l'exécution de la loi (Messanga, 2018a).

Après leurs indépendances, respectivement en 1960 pour le Cameroun sous administration française et en 1961 pour le Cameroun sous administration anglaise, l'ancien British Southern Cameroon a choisi, lors d'un plébiscite organisé par l'ONU en 1961, de se réunir à la République du Cameroun pour former une fédération de deux États de statuts égaux. Ainsi, le bilinguisme officiel ou le biculturalisme linguistique camerounais est la conséquence de l'héritage colonial de ce pays. Mais, il n'est pas sans poser des problèmes de relations intergroupes. En effet, la fédération, considérée comme une fin en soi par les autorités anglophones, était conçue par les gouvernants francophones comme une phase préparatoire voilée pour l'assimilation totale 
du Cameroun méridional dans un État unitaire hautement centralisé et francophone. C'est dans ce sillage qu'est né le conflit intergroupe linguistique connu sous l'appellation de problème anglophone au Cameroun (Tchinda Kenfo, 2017).

Le concept problème anglophone résume l'oppression dont les Anglophones se disent victimes au Cameroun ${ }^{4}$. Celle-ci se manifesterait par la marginalisation, le manque de considération ou la non prise en compte de la spécificité de la minorité anglophone par un pouvoir aux mains de la majorité francophone depuis 1960 (Fonchingong, 2013 ; Messanga \& Dzuetso Mouafo, 2014). Cette marginalisation détériore la qualité des relations entre les populations anglophones et l'élite dominante francophone. En effet, de nombreux Anglophones affirment qu'ils se sont vus refuser le droit à une véritable autodétermination au moment de l'indépendance, et que les accords fédéraux conclus à l'époque ont été par la suite illégitimement démantelés par le gouvernement central contrôlé par les Francophones (Konings \& Nyamnjoh, 2003). D’après eux, ce gouvernement central privilégierait la population francophone et opterait pour une politique systématique d'assimilation et de marginalisation des Anglophones. Ceux-ci seraient privés de leurs droits et traités avec suspicion ; verraient leurs intérêts négligés et leur participation à la vie nationale limitée par l'occupation de fonctions administratives et politiques non essentielles ; se confronteraient à la violence et la répression suite à leurs revendications ; et assisteraient impuissants à la confiscation de leurs ressources économiques par les Francophones (Bouddih, 2006 ; Konings \& Nyamnjoh, 1997 ; Takougang \& Krieger, 1998). De même, leurs ressources naturelles seraient exploitées de manière scandaleuse et utilisées dans les domaines qui profitent directement ou indirectement aux Francophones. À titre illustratif, la Société Nationale de Raffinage (SONARA), située en zone anglophone, a toujours été dirigée par des Francophones, et les revenus tirés du pétrole, dont les gisements se trouvent en zone anglophone, ne seraient utilisées que pour stimuler le développement des régions francophones du pays (Toh, 2001).

Un autre fait ayant irrité la communauté anglophone est la célébration des 50 ans d'indépendance du pays en 2010. Or, le Cameroun actuel, constitué d'Anglophones et de Francophones, n'a pas accédé à l'indépendance le $1^{\text {er }}$ janvier 1960. C'est le Cameroun français qui a obtenu son indépendance ce jour-là, tandis que la partie anglophone du pays a été déclarée indépendante le 1er octobre 1961, jour de la réunification (Fonchingong, 2013). Par conséquent, cette célébration est considérée par les Anglophones comme une déformation délibérée des faits historiques, dont la finalité est la négation de

${ }^{4}$ Pour parler de ce problème, on utilisera le conditionnel parce que « ce sont précisément les Anglophones qui perçoivent «ce problème », alors que les francophones le minimisent ou l'ignorent. » (Messanga, 2018b : 257) 
leur existence en tant que peuple ayant une histoire. La conséquence de ces postures dominantes des Francophones est que les Anglophones se sentent considérés comme des citoyens de seconde zone, voire comme « une minorité opprimée dont le territoire a été annexé. » (Konings, 1996 : 25)

Ces expériences de marginalisation et de domination sont autant de facteurs qui poussent les Anglophones à exprimer des griefs à l'encontre du système gouvernant. Même si ces griefs semblent reposer sur des éléments objectifs et des preuves juridiques solides, il demeure que les revendications qui en découlent se heurtent à un certain nombre d'obstacles qui constituent une entrave dans la lutte contre l'oppression dont ils se disent victimes. Ces obstacles sont relatifs aux stratégies de résistance du régime gouvernant ; au manque de leadership crédible et engagé qui se manifeste par l'existence de nombreux groupes non coordonnés; aux objectifs contradictoires ; à une stratégie inappropriée ; à l'absence de facteurs unificateurs chez les Anglophones ; et à la rivalité entre groupes anglophones (Ngoh, 1996). Ces obstacles facilitent l'utilisation par le régime de diverses stratégies de résistance et de perpétuation de l'oppression à l'égard des membres de cette communauté linguistique, telles que la banalisation et la répression. Au cours de ces dernières années, les tensions se sont sérieusement intensifiées et, depuis novembre 2016, une crise ayant des relents sécessionnistes a éclaté, générant des atteintes aux droits de l'Homme autant chez les forces de sécurité que chez les membres des groupes séparatistes armés qui revendiquent l'indépendance des régions anglophones du Cameroun.

Les causes et circonstances du déclenchement des conflits sont nombreuses et variées (Abdel Ghaffar \& Sorbo, 1990). Cependant, une fois déclenchés, ils sont indifférenciables quant à leurs conséquences. Ceci est dû à la logique délibérément destructrice de tout conflit ; chacune des parties cherchant à infliger le plus grand dommage à l'autre. La crise en cours dans les régions anglophones du Cameroun ne déroge pas à cette règle. On y observe l'escalade de la violence caractérisée par des homicides aveugles ; la détérioration des conditions de vie des populations ; la destruction de certains villages par les séparatistes ou les forces de sécurité ; les enlèvements, ainsi que des assassinats des civils et des militaires (Tchinda Kenfo, 2017). Selon International Crisis Group (2019), en vingt mois, le conflit a fait 1850 morts, 530000 déplacés internes et des dizaines de milliers de réfugiés. Sur le plan social, les infrastructures économiques et sociales de cette zone ont été sérieusement endommagées et dégradées; limitant ainsi ses capacités à répondre aux besoins de base de la population (éducation, santé et services administratifs). Il en découle qu'après ce conflit, l'effort de reconstruction et de réhabilitation social sera considérable. Les dégâts de ce conflit à long terme concernent la désintégration de l'organisation socioculturelle des communautés due aux déplacements des populations pendant le conflit, les 
traumatismes psychologiques infligés aux survivants et la dégradation des écosystèmes. Or, dans ce type de situation, les dégâts à long terme sont souvent négligés, en raison du fait qu'ils ne constituent pas des événements observables immédiatement (la destruction d'un hôpital par exemple), mais plutôt des processus qui ne se concrétiseront qu'après une longue période.

Le problème anglophone est, comme précisé plus haut, causé par l'oppression (la marginalisation et les inégalités sociales) dont les Anglophones se disent victimes. En effet, non seulement ils relèvent que les ressources les plus prestigieuses et les positions politico-administratives de premier plan sont réservées aux seuls Francophones, mais aussi ils notent que ces derniers utilisent la force répressive dont ils ont le contrôle pour maintenir le calme et la stabilité lorsque les Anglophones dénoncent la marginalisation et des injustices sociales dont ils s'estiment victimes (Bouddih, 2006). Ainsi, Le groupe francophone est dans une posture de domination, et ses membres sont témoins de la répression qui a cours dans les régions anglophones depuis le déclenchement de la crise en cours depuis novembre 2016.

En lien avec la théorie des émotions analysée plus haut, la présente étude suggère que les Francophones pourraient ressentir des émotions collectives; ce d'autant plus qu'ils appartiennent au groupe que l'on peut considérer comme l'agresseur, puisqu'il est réputé avoir longtemps marginalisé et infligé des peines à l'exogroupe anglophone. Cette suggestion découle du lien théorique établi entre conflits intergroupes, marginalisation, passé colonial et culpabilité collective, une réponse émotionnelle largement liée à de tels événements (Licata \& Klein, 2005 ; 2010). Dans les faits, les individus peuvent éprouver de la culpabilité collective en relation avec des transgressions historiques entre groupes et des privilèges socio-économiques illégitimes (ou considérés comme tels) accumulés au détriment de l'exogroupe (Leach et al., 2006). Ce sentiment de culpabilité collective motive les membres des groupes dominants à soutenir les excuses et les actions compensatoires des souffrances infligées aux membres des exogroupes dominés qui en sont les victimes (Allpres et al., 2010). C'est cette thèse qui est mise à l'épreuve dans la présente étude, dont l'objectif est d'examiner le lien hypothétique entre la culpabilité groupale que pourraient ressentir les Francophones et leur soutien à la réparation de l'oppression à l'égard des Anglophones.

\section{Méthode}

\section{A. Participants}

Les participants sont 184 étudiants des deux sexes fréquentant diverses facultés de 1'Université de Dschang (Cameroun), sélectionnés par la méthode d'échantillonnage au hasard simple. Ils appartiennent tous à la communauté linguistique francophone, car l'étude mesure l'émotion groupale des membres de cette communauté à l'égard des violences que leurs élites infligent à la 
minorité anglophone. Leur âge est compris entre 18 et 50 ans $(\mathrm{M}=21.91$; É.T.=4.59). Ils ont tous marqué leur accord pour participer bénévolement à l'étude. Du point de vue de l'éthique de la recherche, des garanties leur ont été données quant à l'usage qui serait fait des informations qu'ils fourniraient dans le cadre de l'étude.

\section{B. Matériel et Procédure}

La méthodologie de cette recherche s'est inspirée de l'étude d'Allpress et al. (2010). Ainsi, pour évaluer la culpabilité groupale ressentie, les items utilisés par ces auteurs ont été reformulés et adaptés au contexte des relations intergroupes linguistiques au Cameroun. Par exemple, l'item 1 propose que : « J'éprouve de la culpabilité lorsque j'observe les atrocités et les violences que le gouvernement camerounais fait endurer aux Anglophones du Nord-ouest et du Sud-ouest. » L'item 2 indique que : « Je ressens de la culpabilité en raison de la manière dont les anglophones du Nord-ouest et Sud-ouest sont actuellement traités par le gouvernement camerounais. » Dans le cadre de la présente étude, cet instrument de mesure présente un indice de consistance interne satisfaisant $(\alpha=.74)$.

L'instrument utilisé pour mesurer le soutien à la réparation de l'oppression à l'égard de la minorité anglophone du Cameroun est constitué de 10 items inspirés de la littérature relative aux stratégies de réparation de la violence intergroupe (Staub, 2008). Il évalue précisément cinq aspects des stratégies de réparation de la violence intergroupe. Le premier concerne le soutien à la signature des pétitions contre l'oppression de la minorité anglophone. Il comprend deux (2) items, dont le premier est formulé comme suit : «Les camerounais devraient tous s'engager dans la signature des pétitions (plaintes) contre les violences qui ont cours présentement dans la zone anglophone. » Le deuxième évalue le soutien à la reconstruction des édifices publics. Il comporte quatre (4) items, dont le premier est formulé de la manière suivante : «Il serait encourageant que le gouvernement camerounais reconstruise les bâtiments administratifs dans la zone anglophone. » Le troisième mesure le soutien à la compensation financière. Son unique item indique que: «Je soutiens l'idée selon laquelle le gouvernement camerounais doit compenser financièrement les dégâts causés dans la zone anglophone. » Le quatrième évalue le soutien à la reprise de l'école par les élèves et étudiants. Il a trois (3) items. Le premier est formulé de la manière suivante : «Le gouvernement camerounais devrait réhabiliter les établissements scolaires détruits lors des violences dans la zone anglophone. \) Le cinquième s'intéresse au soutien à la réconciliation avec la minorité anglophone. Il a deux (2) items. Le premier propose que : «Le gouvernement camerounais devrait rechercher la réconciliation avec les populations anglophones du pays. » Cet instrument présente également un 
indice de consistance interne satisfaisant $(\alpha=.82)$. Les participants devaient indiquer leur degré d'accord ou de désaccord pour chacun des items des deux instruments utilisés sur une échelle de type Likert en 7 points, allant de 1 (fortement en désaccord) à 7 (fortement en accord).

\section{Résultats}

Les résultats de cette étude sont présentés en deux temps. Tout d'abord, on analyse les statistiques descriptives et les corrélations. Les premières ont pour but de ressortir une distribution des tendances observées pour chaque variable/dimension. Les secondes, par le biais des indices d'associations linéaires $(r)$ y relatives, permettent d'évaluer la force des liens entre les variables de l'étude et leurs dimensions respectives. Ensuite, on rend compte de l'analyse de la régression entre la culpabilité groupale ressentie et le soutien à la réparation de l'oppression intergroupe. Cette méthode statistique permet d'examiner la relation de causalité entre ces deux variables quantitatives, en mettant en lumière le poids de l'effet de la première (variable indépendante) sur la seconde (variable dépendante). Ce poids s'analyse concrètement grâce aux coefficients standardisés bêta $(\boldsymbol{\beta})$ et aux valeurs $t$ de bêta.

\section{A. Analyse des statistiques descriptives et des corrélations linéaires}

Tableau 1 : Statistiques descriptives et corrélationnelles entre la culpabilité groupale ressentie et le soutien à la réparation de l'oppression à l'égard de la minorité anglophone du Cameroun

\begin{tabular}{|c|c|c|c|c|c|c|c|c|}
\hline Mesure & Moyenne & $\begin{array}{l}\text { Écart- } \\
\text { type }\end{array}$ & 2 & 3 & 4 & 5 & 6 & 7 \\
\hline $\begin{array}{l}\text { 1) Culpabilité } \\
\text { groupale ressentie }\end{array}$ & 9,45 & 3,400 &, $468^{* *}$ &, $148^{*}$ & , 144 &, $159^{*}$ & ,134 &, $311^{\text {*** }}$ \\
\hline $\begin{array}{l}\text { 2) Soutien à la } \\
\text { signature des pétitions }\end{array}$ & 9,86 & 3,041 & - &, $354^{* * *}$ &, $378^{* * *}$ &, $398^{* * *}$ &, $227^{* *}$ &, $695^{* *}$ \\
\hline $\begin{array}{l}\text { 3) Soutien à la } \\
\text { reconstruction des } \\
\text { édifices publics }\end{array}$ & 17,32 & 3,495 & & - &, $481^{* * *}$ &, $566^{* * *}$ &, $409^{* *}$ &, $827^{\text {*** }}$ \\
\hline $\begin{array}{l}\text { 4) Soutien à la } \\
\text { compensation } \\
\text { financière }\end{array}$ & 5,20 & 1,758 & & & - &, $504^{* *}$ &, $309^{* *}$ &, $699^{* * *}$ \\
\hline $\begin{array}{l}\text { 5) Soutien à la reprise } \\
\text { de l'école }\end{array}$ & 11,68 & 2,254 & & & & - &, $276^{* *}$ &, $759^{* * *}$ \\
\hline $\begin{array}{l}\text { 6) Soutien à la } \\
\text { réconciliation }\end{array}$ & 12,38 & 1,895 & & & & & - &, $574^{* * *}$ \\
\hline $\begin{array}{l}\text { 7) Soutien général à } \\
\text { la réparation de } \\
\text { l'oppression } \\
\text { intergroupe }\end{array}$ & 56,43 & 9,030 & & & & & & - \\
\hline
\end{tabular}

Note : **. La corrélation est significative au niveau 0.01 (bilatéral). *. La corrélation est significative au niveau 0.05 (bilatéral); avec $\mathrm{N}=184$. 
Le tableau 1 indique que d'un point de vue descriptif, les participants ressentent de la culpabilité groupale $(\mathrm{M}=9.45>7$; É. $-\mathrm{T}$. $=3.4)$. Ils soutiennent également la réparation de l'oppression à l'égard de la minorité linguistique anglophone $(\mathrm{M}=56.43>35$; É.-T. =9.03). Dans les détails, on observe les mêmes tendances pour les différents aspects de la mesure du soutien à la réparation de l'oppression à l'égard de cette minorité linguistique. Cela signifie qu'en plus d'éprouver de la culpabilité groupale, les participants soutiennent les stratégies de réparation de l'oppression. Pour ce qui concerne les analyses corrélationnelles, ce tableau présente un lien positif et significatif entre la culpabilité groupale ressentie par les participants et le soutien à la réparation de l'oppression à l'égard de la minorité anglophone ( $r=.311 ; p$ $<.01)$. Une analyse détaillée indique que les cinq dimensions du soutien à la réparation de l'oppression intergroupe sont positivement et significativement corrélées entre elles (indices allant de $r=.27 ; p<.01$ à $r=.56 ; p<.01$ ) et avec le construit global (indices allant de $r=.574 ; p<.01$ à $r=.82 ; p<.01$ ). Ces cinq aspects sont également positivement corrélés avec le sentiment de culpabilité groupale $(r=.46 ; p<.01$ pour la corrélation avec le soutien à la signature des pétitions $; r=.14 ; p<.05$ pour la corrélation avec le soutien à la reconstruction des édifices publics $; r=.14 ; p>.05$ pour la corrélation avec le soutien à la compensation financière $; r=.15 ; p<.05$ pour la corrélation avec le soutien à la reprise de l'école ; et $r=.13 ; p>.05$ pour la corrélation avec le soutien à la réconciliation). En somme, ces statistiques révèlent que plus les Francophones éprouvent de la culpabilité groupale, plus ils sont enclins à soutenir la réparation de l'oppression à l'égard de de la minorité anglophone. Les analyses de la régression permettent d'examiner plus finement les effets prédicteurs de chaque variable.

\section{B. Analyse de la régression linéaire}

Tableau 2 : Statistiques de régression entre la culpabilité groupale ressentie et le soutien à la réparation de l'oppression à l'égard de la minorité Anglophone

\begin{tabular}{|c|c|c|c|c|c|c|}
\hline Mesure & $R^{2}$ & $\beta$ & Sig. & Bêta & $t$ & Sig. \\
\hline $\begin{array}{l}\text { 2) Soutien à la signature des } \\
\text { pétitions }\end{array}$ & ,219 & ,215 & ,000 & ,468 & 10,042 &, 000 \\
\hline $\begin{array}{l}\text { 3) Soutien à la reconstruction } \\
\text { des édifices publics }\end{array}$ & ,022 & ,017 & ,045 & , 148 & 20,992 & ,000 \\
\hline $\begin{array}{l}\text { 4) Soutien à la compensation } \\
\text { financière }\end{array}$ & ,021 & ,015 & ,051 & , 144 & 11,817 & ,000 \\
\hline $\begin{array}{l}\text { 5) Soutien à la reprise de } \\
\text { l'école }\end{array}$ & ,025 & ,020 & ,031 & , 159 & 21,936 & ,000 \\
\hline $\begin{array}{l}\text { 6) Soutien à la réconciliation } \\
\text { 7) Soutien général à la }\end{array}$ & ,018 & ,013 & ,069 & ,134 & 28,400 &, 000 \\
\hline $\begin{array}{l}\text { réparation de l'oppression } \\
\text { intergroupe }\end{array}$ & ,097 & ,092 & ,000 & ,311 & 25,896 & ,000 \\
\hline
\end{tabular}

Note : Prédicteurs : (Constante), Culpabilité groupale ressentie 
Ce tableau présente les différents effets de la culpabilité groupale ressentie sur le soutien à la réparation de l'oppression intergroupe et ses différentes modalités. Concernant le soutien à la signature des pétitions, l'analyse de la régression indique un effet positif et significatif de la culpabilité groupale ressentie $(\beta(1841)=.215 ; p<.01$ et $t(1841)=10,042 ; p<.01)$. Cet effet est également observé pour le soutien à la reconstruction des édifices publics $(\beta(1841)=.017 ; p<.05$ et $t(1841)=20,992 ; p<.01) ;$ pour le soutien à la reprise de l'école $(\beta(1841)=.020 ; p<.05$ et $t(1841)=21,936$; $p<.01)$ et pour le soutien à la réparation de l'oppression intergroupe $(\beta$ (184 $1)=.092 ; p<.01$ et $t(1841)=25,896 ; p<.01)$. On note par ailleurs des effets positifs, mais non significatifs, entre la culpabilité groupale ressentie et, d'une part, le soutien à la compensation financière $(\beta(1841)=.015 ; p>.05$ et $t(1841)=11,817 ; p<.01)$ et le soutien à la réconciliation d'autre part $(\beta$ $(1841)=.013 ; p>.05$ et $t(1841)=28,4 ; p<.01)$. Ainsi, bien que certains effets soient non significatifs, la tendance générale présente des effets positifs. On peut en conclure qu'au Cameroun, la culpabilité groupale ressentie par les Francophones détermine leur soutien à la réparation de l'oppression à l'égard des Anglophones. Ces résultats apportent un soutien empirique à l'hypothèse mise à l'épreuve dans la présente recherche.

\section{Discussion}

La présente recherche se situe dans la perspective des travaux qui établissent un lien entre la culpabilité groupale ressentie et les attitudes prosociales, comme le soutien aux stratégies de réparation de l'oppression intergroupe (Iyer \& Leach, 2008 ; Thomas et al., 2009). Elle a mis à l'épreuve l'hypothèse que la culpabilité groupale ressentie détermine le soutien des individus à la réparation de l'oppression intergroupe. De manière spécifique, on s'attendait à ce qu'en contexte camerounais, la culpabilité groupale ressentie par les membres de la majorité linguistique francophone détermine leur soutien à la réparation de l'oppression à l'égard de la minorité linguistique anglophone. Les données collectées à l'issue de cette recherche apportent un soutien empirique à cette hypothèse. En effet, elles indiquent que la culpabilité groupale ressentie par les participants affecte positivement leur soutien à la réparation de l'oppression à l'égard de l'exogroupe anglophone. Ainsi, ces résultats contribuent à l'extension des observations d'Allpress et al. (2010), pour qui les émotions collectives ressenties par les membres d'un groupe sont à l'origine de leur réaction à l'égard des injustices commises par l'endogroupe à l'endroit d'un exogroupe victime.

Dans la même veine que les résultats de la présente recherche, des études se situant dans le domaine de la culpabilité collective rapportent que celle-ci a un effet sur les intentions abstraites et comportementales de réparation de l'oppression (Doosje et al., 1998 ; Iyer et al., 2007 ; Leach et al., 
2006 ; McGarty et al., 2005). Cette tendance pourrait être motivée par une prise de conscience du fait que les membres de l'exogroupe victime souffrent à cause des actes de l'endogroupe. En accord avec cette thèse, Brown et Čehajić (2008) affirment que l'empathie est un médiateur important pour expliquer l'effet de la culpabilité collective sur les stratégies de réparation. Ces auteurs sont convaincus que la relation entre la culpabilité et les stratégies compensatoires est expliquée par le niveau d'empathie des répondants vis-àvis de l'exogroupe. Cette culpabilité collective aide à rétablir des relations intergroupes harmonieuses.

Les résultats de cette recherche confirment le fait que la culpabilité collective est liée à divers comportements et attitudes prosociaux, comme le soutien aux excuses officielles du gouvernement pour des mauvais traitements historiques (McGarty et al., 2005), le désir pour un contact positif avec les victimes, la volonté d'adopter des stratégies pour réduire l'exclusion sociale (Brown et al., 2008) et la diminution des préjugés (Iyer et al., 2003). Concrètement, cela signifie que les participants de cette étude éprouvent le désir de restaurer leur image groupale menacée par les conduites discriminatoires et répressives des élites de leur communauté linguistique à l'endroit de la minorité anglophone. De nombreux exemples de ce type sont relevés dans la littérature. Nobles (2008) rapporte ainsi qu'en 1995, la reine Elizabeth II d'Angleterre et le Premier Ministre néo-zélandais se sont excusés auprès de la communauté Maori pour avoir violé le traité de Waitangi. De même, en 1993, le Président Américain Bill Clinton s'est excusé auprès des Hawaïens pour avoir participé à la violation de leur souveraineté. Au cours de la même année, le gouvernement canadien s'est excusé auprès des populations indigènes pour la maltraitance historique qu'elles ont subie. D'après Staub (2008), cette disposition attitudinale est l'une des alternatives efficaces pour éviter les griefs ressentis et les possibilités d'une éventuelle planification de vengeance à court ou à long terme de la part des personnes appartenant aux groupes victimes de l'oppression des membres des groupes de statut fort. Elle est aussi efficace dans la mesure où elle contribue à la réduction du sentiment d'impuissance et de la perception de la vulnérabilité de l'endogroupe chez les personnes victimes de l'oppression sociale, parce qu'elle les place dans une position où elles résisteront à la tentation d'entretenir le cycle de la violence, en posant des actes de vengeance.

\section{Conclusion}

La présente étude avait pour objectif de vérifier, en contexte camerounais, le lien entre la culpabilité groupale ressentie par les Francophones et leur soutien à la réparation de l'oppression à l'égard des Anglophones. Au plan théorique, elle tire sa source des travaux d'Allpress et al. (2010) qui défendent l'idée que les émotions collectives ressenties par les 
membres des groupes dominants les poussent à soutenir les excuses et les actions compensatoires des souffrances infligées aux membres des exogroupes dominés qui en sont les victimes. Les résultats obtenus à l'issue de la collecte des données ont permis de confirmer l'hypothèse formulée. Ils se situent donc dans le continuum des observations rapportées par les études qui révèlent que la culpabilité collective pousse les membres de groupes dominants à adopter des comportements et attitudes prosociaux à l'égard des exogroupes victimes de mauvais traitements historiques (Brown \& Čehajić, 2008 ; Iyer et al., 2003 ; McGarty et al., 2005).

\section{References:}

1. Abdel Ghaffar, M. A., \& Sorbo, J. (1990). Managment of crisis in the Sudan, centre for development studies. Norway: University of Bergen.

2. Abwa, D. (2000). Le problème anglophone au Cameroun : facteur d'intégration ou de désintégration nationale? in C. Dubois, M. Michel, \& P. Soumille (éds), Frontières plurielles, frontières conflictuelles en Afrique subsaharienne (pp. 115-141). Paris: L'Harmattan.

3. Allpress, A. J., Barlow, F. K., Brown, R., \& Louis, W. R. (2010). Atoning for Colonial Injustices: Group-Based Shame and Guilt Motivate Support for Reparation. International Journal of Conflit and Violence, 4 (1), 75-88.

4. Avruch, K. \& Vejerano, B. (2002). Truth and reconciliation commissions: A review essay and annotated bibliography. Online Journal of Peace and Conflict Resolution, 4, 37-76.

5. Bandura, A. (1999). Moral disengagement in the perpetration of inhumanities. Personality and Social Psychology Review, 3, 193-209.

6. Barkan, A. (2001). The guilt of nation: Restitution and negotiating historical injustice. Journal of Social Issues, 46, 65-81.

7. Baumeister, R. F., Stillwell, A. M., \& Heatherton, T. F. (1994). Guilt: An interpersonal approach. Psychological Bulletin, 115 (2), 43-67.

8. Blatz, C. W., Schumann, K., \& Ross, M. (2009). Government apologies for historical injustices. Political Psychology, 30, 219-241.

9. Bouddih, A. (2006). The Post online newspaper. http://www.postnewsline.com/2006/10/focus_on_45th_a.html.

10. Boyes-Watson, C. (2008). Peacemaking circles and urban youth: Bringing justice home. MN : Living Justice Press.

11. Braithwaite, J. (2002). Stetting standards for restorative justice. British Journal of Criminologie, 42, 563-577.

12. Brown, R. \& Čehajić, S. (2008). Dealing with the past and facing the future : Mediators of the effects of collective guilt and shame in Bosnia and Herzegovina. European Journal of Social Psychology, 38 (4), 6984. 
13. Cehajic, S., Brown, R. J., \& Castano, E. (2008). Forgive and forget ? Antecedents and consequences of intergroup forgiveness in Bosnia and Herzegovina. Political Psychology, 29, 351-367.

14. Cross, S. \& Rosenthal, R. (1999). Three models of conflict resolution: Effects on intergroup expectancies and attitudes. Journal of Social Issues, 55, 561-580.

15. Deutsch, M. (1973). The resolution of conflict: Constructive and destructive processes. New Haven: Yale University Press.

16. Doosje, B., Branscombe, N. R., Spears, R., \& Manstead, A. S. R. (1998). Guilty by association: When one's group has a negative history. Journal of Personality and Social Psychology, 75 (4), 72-86.

17. Du Toit, F. \& Doxtader, E. (2010). In the balance: South Africans debate reconciliation. Johannesburg: Jacana Media.

18. Fischer, A. H. \& Manstead, A. S. R. (2016). Social functions of emotion and emotion regulation. In L. F. Barrett., M. Lewis., \& J. M. Haviland-Jones (Eds.), Handbook of emotions. New York: Guilford.

19. Fonchingong, T. (2013). The quest for autonomy: The case of Anglophone Cameroon. African Journal of Political Science and International Relations, 7, 5, 224-236.

20. International Crisis Group (2019). Crise anglophone au Cameroun: comment arriver aux pourparlers. Rapport Afrique $N^{\circ} 272$. Nairobi/Bruxelles, 2 mai 2019.

21. Iyer, A., \& Leach, C. W. (2008). Emotion in intergroup relations. European Review of Social Psychology, 19, 86-125.

22. Iyer, A., Leach, C. W., \& Crosby, F. J. (2003). White guilt and racial compensation: The benefits and limits of self-focus. Personality and Social Psychology Bulletin, 29 (1), 17-29.

23. Iyer, A., Schmader, T., \& Lickel, B. (2007). Why individuals protest the perceived transgressions of their country: The role of anger, shame and guilt. Personality and Social Psychology Bulletin, 33 (4), 72-87.

24. Kelman, H. C. (2008). Reconciliation from a social psychological perspective. In A. Nadler, T. Malloy, \& J. D. Fisher (Eds.), Social psychology of intergroup reconciliation (pp. 15-32). New York: Oxford University Press.

25. Konings, P. (1996). The post-colonial State and Economic and political Reforms in Cameroon. In A.E. Fernandez Jilberto, \& A. Mommen (Eds), Liberalization in the developing world : institutional and economic changes in Latin America, Africa and Asia (pp. 244265). London: Routledge.

26. Konings, P. \& Nyamnjoh, F. B. (2003). Negotiating an Anglophone Identity: A study of the politics of recognition and representation in Cameroon. Afrika-Studiecentrum Series. Leiden: Brill. 
27. Konings, P., \& Nyamnjoh, F. B. (1997). The Anglophone Problem in Cameroon. The Journal of Modern African Studies, 35 (2), 207-229.

28. Kriesberg, L. (2007). Aspects, growth and sequences. International Journal of Peace Studies, 12, 1-22.

29. Lazarus, R. S. (1991). Emotion and Adaptation. New York: Oxford University Press.

30. Leach, C. W., Iyer, A., \& Pederson, A. A. (2006). Anger and guilt about ingroup advantage explain the willingness for political action. Personality and Social Psychology Bulletin, 32 (9), 32-45.

31. Lewis, H. B. (1971). Shame and guilt in neurosis. New York: International Universities Press.

32. Licata, L. \& Klein, O. (2005). Regards croisés sur un passé commun : Anciens colonisés et anciens coloniaux face à l'action belge au Congo. In M. Sanchez-Mazas \& L. Licata (eds), L'Autre: Regards psychosociaux (pp 241-278). Grenoble: Presses Universitaires de Grenoble.

33. Licata, L. \& Klein, O. (2010). Holocaust or benevolent paternalism ? Intergenerational comparisons on collective memories and emotions about Belgium's colonial past. International Journal of Conflit and Violence, 4 (1), 45-57.

34. Lindner, E. (2006). Making enemies: Humiliation and international conflict. London: Praeger.

35. Mackie, D. M., Devos, T., \& Smith, E. R. (2000). Intergroup emotions: Explaining offensive action tendencies in an intergroup context. Journal of Personality and Social Psychology, 79, 602-616.

36. Mackie, D. M., Silver, L. A., \& Smith, E. R. (2004). Intergroup emotions: Emotion as an intergroup phenomenon. In L. Z. Tiedens \& C. W. Leach (Eds.), The social life of emotions (pp. 227-245). Cambridge: Cambridge University Press.

37. Manstead, A. S. R., \& Fischer, A. H. (2001). Social appraisal: The social world as object of and influence on appraisal processes. In K. R. Scherer, A. Schorr, \& T. Johnstone (Eds.), Appraisal Processes in Emotion (pp. 221-232). New York: Oxford University Press.

38. McGarty, C., Pedersen, A., Leach, C. W., Mansell, T., Waller, J., \& Bliuc, A. M. (2005). Group-Based guilt as a predictor of commitment to apology. The British Journal of Social Psychology, 44 (4), 59-80.

39. Messanga, G. A. (2018a). L'évaluation psychométrique de la distinctivité optimale dans les relations intergroupes linguistiques au Cameroun. nkó'Lumière, 19, 85-108.

40. Messanga, G. A. (2018b). Effet de la scolarisation des élèves issus de familles francophones dans le sous-système éducatif anglophone sur l'identité sociale anglophone: une analyse dans la perspective 
théorique de la menace intergroupe. In A. E. Ebongue \& A. Djoum Nkwescheu (dir.), L'insécurité linguistique dans les communautés anglophone et francophone du Cameroun (pp. 251-274). Paris: L'Harmattan.

41. Messanga, G. A. \& Dzuetso Mouafo, A. V. (2014). Contact intergroupe et préjugés à l'égard des anglophones en milieu universitaire bilingue et monolingue francophone au Cameroun. $n k a$, lumière, 12, 179-202.

42. Nadler, A. (2012). Intergroup reconciliation: Definitions, processes and future directions. In L. Tropp (Ed.), The Oxford book of intergroup conflict (pp. 291-309). New York: Oxford University Press.

43. Nadler, A., \& Shnabel, N. (2015). Intergroup reconciliation: Instrumental and socio-emotional processes and the needs-based model. European Review of Social Psychology, 26 (1), 93-125.

44. Ngoh, J. V. (1996). History of Cameroon since 1800. Limbe: Press Book.

45. Nobles, M. (2008). The Politics of official Apologies. New York: Cambridge University Press.

46. Parkinson, B. (2001). Putting appraisal in context. In K. R. Scherer, A. Schorr, \& Johnstone (Eds.), Appraisal processes in emotion (pp. 173-186). New York: Oxford University Press.

47. Rimé, B. (2009). Emotion Elicits the Social Sharing of Emotion: Theory and Empirical Review. Emotion Review, 1, 60-85.

48. Rouhana, N. (2004). Group identity and power asymmetry in reconciliation processes : The Israeli Palestinian case. Peace and Conflict: Journal of Peace Psychology, 10, 33-52.

49. Sherif, M. (1958). Superordinate goals in the reduction of intergroup conflict. American Journal of Sociology, 63, 349-356.

50. Smith, E. R. (1993). Social identity and social emotions: Toward new conceptualizations of prejudice. In D. M. Mackie \& D. L. Hamilton (Eds.), Affect, cognition, and stereotyping : Interactive processes in group perception (pp. 297-315). San Diego, CA : Academic Press.

51. Staub, E. (2008). Promoting reconciliation after genocide and mass killing in Rwanda And other postconflict settings: Understanding the roots of violence, healing, shared History, and general principles. In A. Nadler, T. Malloy, \& J. D. Fisher (Eds.), Social psychology of intergroup reconciliation (pp. 395-422). New York: Oxford University Press.

52. Takougang, J. \& Krieger, M. (1998). African State and Society in the 1990s: Cameroon's Political Crossroads. Boulder-Oxford: Westview Press. 
53. Tam, T., Hewstone, M., Kenworthy, J. B., Cairns, E., Marinetti, C., Geddes, L., \& Parkinson, B. (2008). Postconflict reconciliation: Intergroup forgiveness and implicit biases in Northern Ireland. Journal of Social Issues, 64, 303-320.

54. Tangney, J. P., \& Fischer K. W. (1995). Self conscious emotions: The psychology of shame, guilt, embarrassment and pride. New York : Guilford.

55. Tchinda Kenfo, J. (2017). Le "problème anglophone" au Cameroun : la réponse par le processus participatif au développement territorial. www.thinkingafrica.org.

56. Thomas, E. F., McGarty, C., \& Mavor, K. I. (2009). Transforming "apathy into movement": The role of prosocial emotions in motivating action for social change. Personality and Social Psychology Review, $13(4), 10-33$.

57. Tiedens, L. Z., \& Leach, C. W. (2004). The social life of emotions. Cambridge: Cambridge University Press.

58. Toh, N. P. (2001). The anglophone problem in Cameroon: The prospects for nonviolent transformation, proposal for peaceful transformation of the anglophone problem in Cameroon. Bradford: M. A. University Bradford Press.

59. Weiss-Klayman, N., Hameiri, B., \& Halperin, E. (2020). Group-based guilt and shame in the context of intergroup conflict: The role of beliefs and meta-beliefs about group malleability. Journal of Applied Social Psychology/Early

View. https://onlinelibrary.wiley.com/doi/abs/10.1111/jasp.12651

60. Whitman, J. (2003). Harsh justice: Criminal punishment and the widerning divide between America and Europe. Oxford: Oxford University Press. 